\title{
The preoperative and the postoperative neutrophil-to-lymphocyte ratios both predict prognosis in gastric cancer patients
}

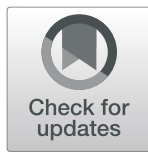

\author{
Eun Young Kim ${ }^{1}$ and Kyo Young Song ${ }^{2^{*}}$ (D)
}

\begin{abstract}
Background: Both the preoperative and postoperative neutrophil-to-lymphocyte ratios (NLRs) have been proposed to predict the long-term prognosis in some cancers, including gastric cancer. The present study investigated the prognostic impact of postoperative NLR, and its preoperative to postoperative changes, in patients with gastric cancer.

Methods: From 2009 to 2012, 1227 consecutive patients who underwent curative surgery for gastric cancer were enrolled in this study. The optimal cut-off value for the postoperative 6-month NLR was 1.7, as determined by receiver operating characteristic curve analysis. Patients were categorized into low- and high-NLR groups based on their postoperative NLR. Four additional groups (low to low, low to high, high to low, and high to high groups) were defined based on the preoperative to postoperative change in the NLR.
\end{abstract}

Results: The 5-year overall survival (OS) rates of the low- and high-NLR group were $90.7 \%$ and $83.0 \%$, respectively $(P<$ 0.001). The differences in OS were significant in stage I and stage III gastric cancer patients $(P<0.001$ and 0.012 , respectively). Postoperative NLR was an independent prognostic factor for OS (hazard ratio $[H R]=1.556 ; P=0.010$ ). The high to high NLR change was a significant predictor of OS (HR $=1.817 ; P=0.003)$.

Conclusions: High preoperative and postoperative NLRs, and especially the persistent elevation of preoperative to postoperative NLR, were significant poor prognostic factors for OS in patients with gastric cancer.

Keywords: Systemic inflammatory response, Postoperative, Neutrophil-to-lymphocyte ratio, Prognostic factor, Gastric cancer

\section{Introduction}

Gastric cancer is the third leading cause of cancerrelated death in men, and the fifth leading cause in women [1]. Gastrectomy with lymph node dissection remains the mainstay treatment for gastric cancer [2] but the prognosis differs among patients. This has led to increased interest in individualized therapy based on the specific characteristics of the tumor. The only reliable prognostic indicator at present is the tumor, node,

\footnotetext{
*Correspondence: skygs@catholic.ac.kr; skys9615@gmail.com

2Department of Surgery, Seoul St. Mary's Hospital, College of Medicine, The Catholic University of Korea, Seoul, Korea

Full list of author information is available at the end of the article
}

metastasis (TNM) stage; however, as patients with the same tumor stage have a heterogeneous prognosis, additional reliable prognostic factors are needed. Accurate prognostic indicators would improve the early management of gastric cancer patients, especially those with a poor prognosis.

Experimental studies have suggested that systemic inflammatory responses play a crucial role in promoting cancer, via pro-inflammatory molecules produced by innate immune cells. There is increasing evidence that the local immune response and systemic inflammation support tumor progression in patients with established cancer, adversely affecting their survival [3]. Recent investigations 
have shown a significant relationship between postoperative inflammatory markers, including C-reactive protein (CRP), leukocytosis, thrombocytosis, and elevated neutrophil-tolymphocyte ratio (NLR) or platelet-to-lymphocyte ratio (PLR), and poor survival in patients with various cancers [4-11]. However, a few studies have examined the association between markers of postoperative inflammation, especially the NLR, and the prognosis of patients with gastric cancer [5, 9-11]. Instead, most studies have focused on preoperative measures [12-18]. Therefore, in this study, we examined the clinical utility of postoperative NLR, and its preoperative to postoperative changes, as prognostic indicators of gastric cancer.

\section{Patients and methods}

Prospectively collected data on 1943 patients with gastric adenocarcinoma who underwent gastrectomy at Seoul St. Mary's Hospital between 2009 and 2012 were reviewed. Of these patients, 1612 patients underwent curative surgery (R0) for gastric cancer. After exclusion, 1227 patients were enrolled in this study. The exclusion criteria were as follows: remnant gastric cancer, neoadjuvant chemotherapy, synchronous and metachronous malignancies, emergency surgery, liver cirrhosis, evidence of a severe inflammatory condition, coexisting hematological malignancies or disorders, autoimmune disorders and recent steroid therapy, incomplete/inaccurate medical records, and absence of postoperative 6-month routine blood examination. Institutional Review Board approval was obtained for this study (KC17RESI0108).

\section{Blood sample analysis}

Blood samples were obtained 6 months postoperatively for determination of the white blood cell, neutrophil, and lymphocyte counts.

\section{Determination of the optimum NLR cut-offs}

The NLR was defined as the neutrophil count divided by the lymphocyte count. The optimal cut-off value for the postoperative 6 -month NLR was 1.7 , as determined by receiver operating characteristic (ROC) curve analysis based on 5-year overall survival (OS) as an endpoint. The area under the ROC curve for the postoperative 6month NLR was 0.563 . Therefore, the sensitivity and specificity of the cut-off value of the NLR for OS were $44.0 \%$ and $73.3 \%$, respectively (Fig. 1). The cut-off value of the preoperative NLR was 2.0. This value was used based on our previous study about the prognostic role of the preoperative NLR in gastric cancer [12]. Patients with an NLR above the cut-off value were assigned to the high-NLR (HNLR) group, and the others were assigned to the low-NLR (LNLR) group. In addition, the patients were categorized into four groups based on the preoperative to postoperative change in the NLR: low to low (LL), low to high (LH), high to low (HL), and high to high $(\mathrm{HH})$ groups.

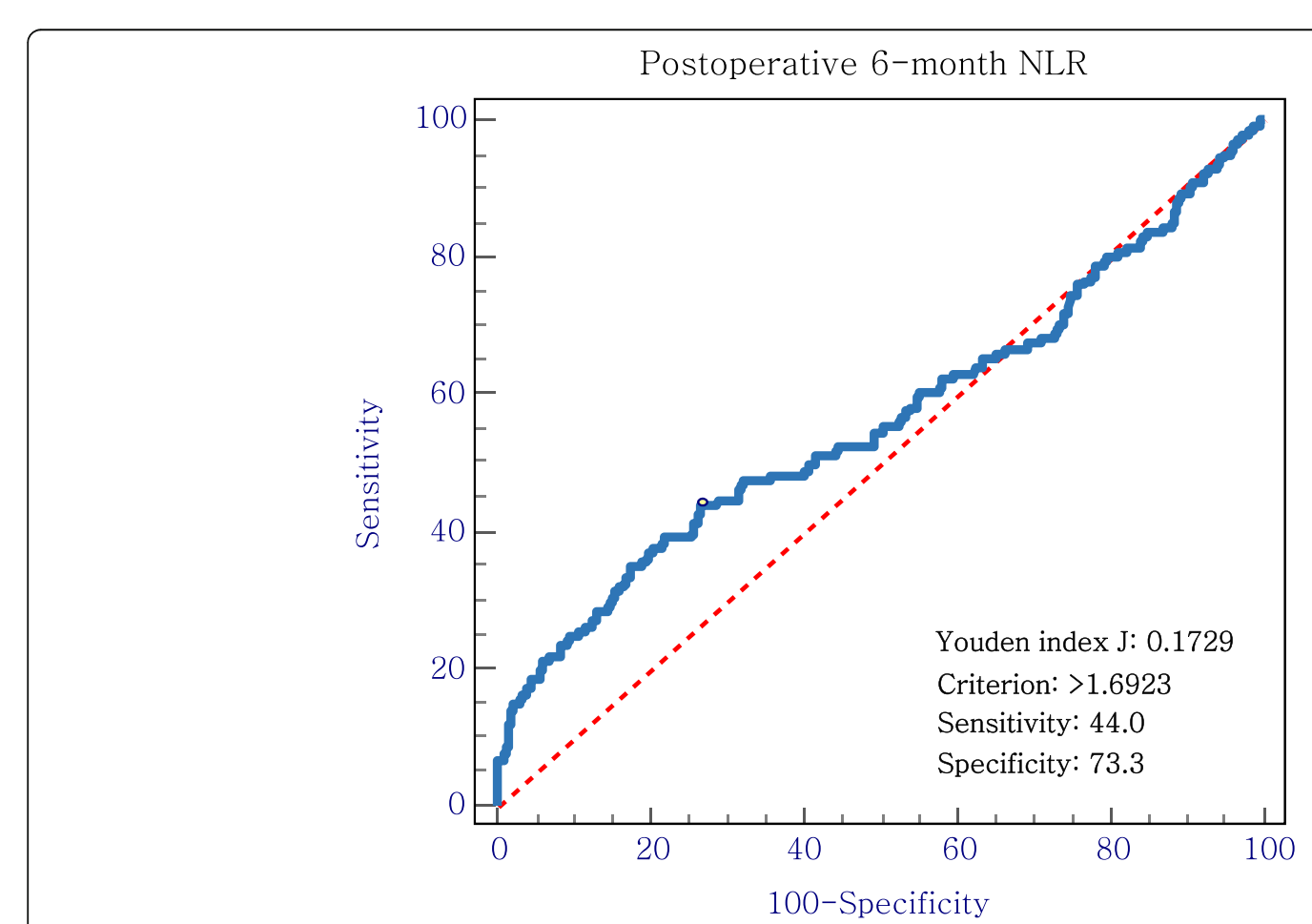

Fig. 1 Receiver operating characteristic curves of the neutrophil to lymphocyte ratio (NLR) in gastric cancer patients at 6 months postoperatively (cut-off value, 1.7; sensitivity, 44.0\%; specificity, 73.3\%) 


\section{Statistical analysis}

ROC curve analysis was performed to evaluate the sensitivity and specificity of the NLR with respect to 5-year OS, and the Youden index was estimated to determine the optimal NLR cut-off values. Categorical variables were compared using the chi-square test. Kaplan-Meier curves were used to estimate OS and disease-free survival (DFS). The groups were compared in terms of survival using the log-rank test. A Cox regression model was used to identify variables influencing OS and DFS. Then multivariate analyses were performed including all variables showing significant independent relationships with OS and DFS. In all analyses, $P<0.05$ was taken to indicate statistical significance. All statistical analyses were performed using the SPSS software (version 18.0; SPSS Inc., Chicago, IL, USA).

\section{Results}

\section{Clinicopathological characteristics}

The 1227 patients enrolled in the study had a mean age of 58.4 years old (SD, 11.8) and 811 (66.1\%) were male. The open approach method was used in $62.2 \%$ cases. Overall, 919 patients (74.9\%) underwent partial gastrectomy, and most of the patients $(n=804$; 65.5\%) presented with stage I disease. There were 649 patients (52.9\%) with undifferentiated-type cancer.

\section{Relationship between clinicopathological characteristics and the inflammation-based score}

Based on the postoperative 6-month NLR cut-off value, there were 875 LNLR and 352 HNLR patients. Older age, male gender, differentiated cancer, and not receiving the adjuvant chemotherapy were significantly associated with a HNLR (Table 1$)(P<0.001, P=0.021,0.002$, and 0.024 , respectively).

\section{Prognostic factors for OS and DFS}

The OS of the HNLR group was significantly lower than that of the LNLR group (Fig. 2a) $(P<0.001)$. In univariate analyses, age, approach method, extent of resection, depth of invasion, node status, stage, adjuvant chemotherapy, preoperative NLR, postoperative NLR, and NLR change were significant prognostic factors for OS. In multivariate analyses, preoperative HNLR, postoperative HNLR, and change in HH NLR were significant independent risk factors for poorer OS after adjusting the inflammatory markers for age, approach method, extent of resection, depth of invasion, node status, stage, and adjuvant chemotherapy (Table 2) $(P=0.025,0.010$, and 0.003, respectively).

In univariate analyses, age, approach method, extent of resection, depth of invasion, node status, stage, adjuvant chemotherapy, preoperative NLR, and NLR change were significant prognostic factors in terms of DFS. In multivariate analyses, preoperative HNLR, postoperative
HNLR, and HH NLR change were not significant risk factors for DFS after adjusting the inflammatory markers for age, approach method, extent of resection, depth of invasion, node status, stage, and adjuvant chemotherapy (Table 3) $(P=0.122,0.513$, and 0.143 , respectively).

\section{Overall survival stratified by tumor stage}

When the OS of the patients were stratified according to tumor stage, HNLR was significantly associated with poor prognosis in stage I and III but not in stage II disease (Fig. 2b, c, d). The 5-year OS rates of the NLR changes were significantly different in all stages (Table 4).

\section{Discussion}

This study examined the associations of postoperative NLR, and its preoperative to postoperative changes, with the prognosis of gastric cancer patients, as well as the clinical utility of these measurements. The results showed that postoperative NLR and changes in NLR are independent prognostic factors for OS in patients with gastric cancer.

Increasing evidence supports an association between cancer and inflammation [19]. In particular, inflammatory processes contribute to cancer initiation, promotion, progression, and invasion [20]. In addition, inflammation is one of the seven main characteristics of cancer [21]. However, the precise mechanism underlying the association between increased NLR and poor long-term outcome in cancer patients is unclear. As NLR depends on two factors (neutrophil and lymphocyte counts), a high NLR may contribute to postoperative prognosis through the following possible mechanism. First, tumor-associated neutrophils may play a role in cancer progression by releasing factors that modulate the extracellular matrix and inflammation in the tumor microenvironment [22]. In particular, they play important roles during the initial angiogenic process in experimental tumor models [23]. Second, lymphocytes are the immune cells most responsible for the body's protective effector immune response and antitumor response. That is, a decrease in circulating lymphocytes indicates a reduction of immune surveillance, thus enabling tumor growth [24]. The results of our clinical study support previous basic research in the same area.

The main strength of this study was its use of reliable NLR cut-off values, based on a large pool of data for gastric cancer patients. In addition, because a complete blood count is routinely obtained in all cancer patients during follow-up, evaluation of the prognosis of these patients required no additional effort. Thus, our method based on measurements of a systemic inflammatory parameter is a simple, cost-effective, and reproducible technique for assessing the survival of gastric cancer patients. Although the present study had a similar setting to a previous study of preoperative NLR [12], our analysis focused on postoperative NLR and dynamic changes in NLR. It is useful to evaluate the postoperative 
Table 1 Associations of patient characteristics with the postoperative neutrophil-to-lymphocyte ratio (NLR)

\begin{tabular}{|c|c|c|c|}
\hline \multirow[t]{2}{*}{ Factors } & \multicolumn{2}{|l|}{ NLR } & \multirow[t]{2}{*}{$P$ value } \\
\hline & LNLR $(n=875)(\%)$ & HNLR $(n=352)(\%)$ & \\
\hline Age in years, (mean $\pm S D$ ) & $57.5 \pm 11.7$ & $60.8 \pm 11.7$ & $<0.001$ \\
\hline Gender & & & 0.021 \\
\hline Male & $561(64.1)$ & $250(71.0)$ & \\
\hline Female & $314(35.9)$ & $102(29.0)$ & \\
\hline Approach method & & & 0.636 \\
\hline Open & $546(62.4)$ & $217(61.7)$ & \\
\hline Laparoscopy & $310(35.4)$ & $120(36.9)$ & \\
\hline Robot & $19(2.2)$ & $5(1.4)$ & \\
\hline Extent of resection & & & 0.173 \\
\hline Partial gastrectomy & $646(73.8)$ & $273(77.6)$ & \\
\hline Total gastrectomy & $229(26.2)$ & 79 (22.4) & \\
\hline Histologic type & & & 0.002 \\
\hline Differentiated & $388(44.3)$ & $190(54.0)$ & \\
\hline Undifferentiated & $487(55.7)$ & $162(46.0)$ & \\
\hline Depth of invasion ${ }^{a}$ & & & 0.428 \\
\hline $\mathrm{T} 1$ & $544(62.2)$ & $219(62.2)$ & \\
\hline $\mathrm{T} 2$ & $87(9.9)$ & $45(12.8)$ & \\
\hline T3 & $119(13.6)$ & $41(11.7)$ & \\
\hline $\mathrm{T} 4$ & $125(14.3)$ & $47(13.3)$ & \\
\hline Node status ${ }^{a}$ & & & 0.141 \\
\hline NO & $588(67.2)$ & $241(68.5)$ & \\
\hline N1 & 119 (13.6) & $35(9.9)$ & \\
\hline N2 & $89(10.2)$ & $33(9.4)$ & \\
\hline N3 & $79(9.0)$ & $43(12.2)$ & \\
\hline Stage ${ }^{a}$ & & & 0.282 \\
\hline 1 & $567(64.8)$ & $237(67.3)$ & \\
\hline$\|$ & $157(17.9)$ & $50(14.2)$ & \\
\hline III & $151(17.3)$ & $65(18.5)$ & \\
\hline Adjuvant chemotherapy & & & 0.024 \\
\hline No & $602(68.8)$ & $265(75.3)$ & \\
\hline Yes & $273(31.2)$ & $87(24.7)$ & \\
\hline
\end{tabular}

$S D$ standard deviation

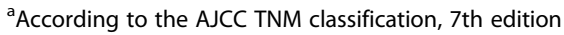

NLR because it may reflect residual host immune activity [25]. The postoperative systemic inflammatory state plays an important role in preventing tumor recurrence. That is, a postoperative increase in NLR indicates a pro-tumor inflammatory response of the host, whereas a postoperative decrease shows an anti-tumor immune response of the host [6]. For clinicians, therapeutic decision-making during the routine follow-up of high-risk patients after surgery is challenging, where early intervention is crucial to prevent recurrence. In clinical fields, tumor markers such as CEA, CA 19-9, and CA 72-4 are widely evaluated as laboratory follow-up tools. However, there are certain limitations to using these markers to detect recurrence and predict survival after surgery, because these markers have low sensitivity. Marrelli et al. reported sensitivities of $44 \%$ for CEA, $56 \%$ for CA $19-9$, and $51 \%$ for CA $72-4$ in patients with recurrence [26]. However, the use of postoperative NLR and changes in NLR combined with existing tumor markers may provide a novel postoperative risk stratification tumor marker model.

The prognostic role of preoperative inflammatory markers has been well studied in gastric cancer patients [12-18]. However, the prognostic role of postoperative NLR in such patients has attracted less research attention 

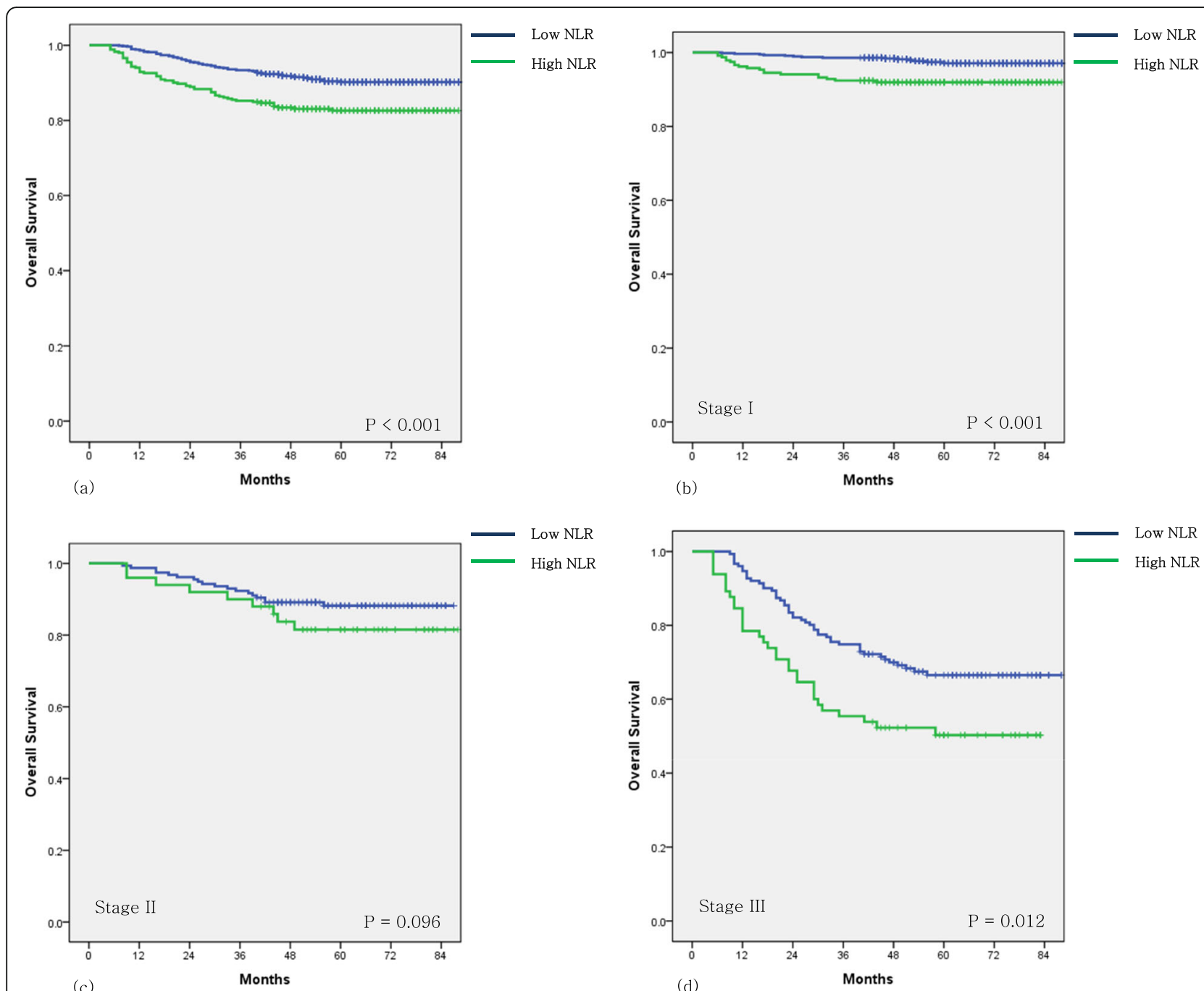

Fig. 2 Survival analysis. a Overall survival (OS) according to the 6-month postoperative NLR $(P<0.001)$. b Patients with stage I gastric cancer: OS according to the NLR at 6 months postoperatively $(P<0.001)$. c Patients with stage II gastric cancer: OS according to the NLR at 6 months postoperatively $(P=0.096)$. d Patients with stage III gastric cancer: OS according to the NLR at 6 months postoperatively $(P=0.012)$

[5, 9-11]. Some researchers have evaluated the prognosis based on the simple postoperative value $[5,11]$, while others have evaluated it according to preoperative to postoperative changes in NLR $[9,10]$. The prognostic importance of this change reflects a dynamic change in the balance between host inflammatory and immune responses rather than the simple preoperative or postoperative NLRs. In Korea, a few studies have examined the associations between postoperative inflammatory markers, especially the NLR, and the prognosis of patients with gastric cancer. Kim et al. suggested that postoperative NLR predicts long-term recurrence after gastric cancer surgery. However, they used the postoperative day 3 NLR value and did not examine the dynamic change from preoperative to postoperative NLR, unlike our study [5]. Min et al. studied changes in NLR, similar to our study, but they divided the patients into two groups, i.e., negative and positive groups, according to the difference between preoperative and postoperative values. In addition, the postoperative NLR evaluation was performed at 3 to 6 months after surgery [10]. To the best of our knowledge, this is the first study to demonstrate the prognostic value of both the postoperative NLR and NLR change in Korean gastric cancer patients. Another strength of our study was that these two prognostic indicators were identified using the same data.

Despite its strengths, our study also had some limitations. First, we performed retrospective analysis of data collected from a single institution. Second, some bias can occur, because our study enrolled the patients even if there was recurrence within 6 months after operation. Thus, authors tried to perform additional analyses 
Table 2 Univariate and multivariate analyses of factors predicting overall survival

\begin{tabular}{|c|c|c|c|c|}
\hline & \multicolumn{2}{|l|}{ Univariate analysis } & \multicolumn{2}{|l|}{ Multivariate analysis } \\
\hline & $\mathrm{HR}(95 \% \mathrm{Cl})$ & $P$ value & Adjusted $\mathrm{HR}^{\mathrm{a}}(95 \% \mathrm{Cl})$ & $P$ value \\
\hline \multicolumn{5}{|l|}{ Preoperative NLR } \\
\hline LNLR $(N L R<2)$ & Reference & & Reference & \\
\hline HNLR (NLR $\geq 2)$ & $1.719(1.306-2.262)$ & 0.000 & $1.449(1.048-2.004)$ & 0.025 \\
\hline \multicolumn{5}{|l|}{ Postoperative NLR } \\
\hline LNLR $(N L R<1.7)$ & Reference & & Reference & \\
\hline HNLR (NLR $\geq 1.7)$ & $1.946(1.410-2.686)$ & 0.000 & $1.556(1.112-2.176)$ & 0.010 \\
\hline \multicolumn{5}{|l|}{ NLR change } \\
\hline LL (preoperative NLR $<2$, postoperative $N L R<1.7$ ) & Reference & & Reference & \\
\hline LH (preoperative NLR $<2$, postoperative NLR $\geq 1.7$ ) & $1.403(0.791-2.490)$ & 0.247 & $1.040(0.575-1.882)$ & 0.896 \\
\hline $\mathrm{HL}$ (preoperative NLR $\geq 2$, postoperative $N L R<1.7$ ) & $1.370(0.894-2.100)$ & 0.148 & $1.070(0.692-1.655)$ & 0.761 \\
\hline $\mathrm{HH}$ (preoperative $\mathrm{NLR} \geq 2$, postoperative $N L R \geq 1.7$ ) & $2.600(1.763-3.834)$ & $<0.001$ & $1.817(1.220-2.706)$ & 0.003 \\
\hline
\end{tabular}

HR hazard ratio, $C l$ confidence interval, NLR neutrophil-to-lymphocyte ratio, LNLR low NLR, HNLR high NLR

${ }^{a}$ Adjusted for age, approach method, extent of resection, depth of invasion, node status, stage, and adjuvant chemotherapy

excluding recurrence within 6 months after operation. The results were shown in Additional file 1: Supplement table. The trend was similar with our original study described in the "Result" section except for non-significant $P$ value of the preoperative NLR in multivariate analysis for OS. Third, we did not evaluate changes in other inflammatory markers such as CRP, procalcitonin, PLR, and neutrophil-to-platelet ratio (NPR). Further studies are needed to validate the significance of our postoperative risk stratification model including NLR and other inflammatory markers, including CRP, procalcitonin, PLR, and NPR. Fourth, our study only demonstrated the postoperative 6-month NLR after surgery due to its retrospective nature. There is no consensus regarding the appropriate timing for measurement of postoperative inflammatory markers as prognostic predictors, with time points to check postoperative NLR ranging from 3 days to 6 months in previous gastric cancer studies $[5,9$, 10]. Surgery is a major event that induces an acute inflammatory response [27]. The acute inflammatory reaction caused by surgery resolves within a short time, and its effects on the tumor disappear completely by several months postoperatively. Although there have been no reports regarding the exact time when inflammation due to the surgical wound healing process ceases, several groups have suggested that 1 month postoperatively is an appropriate check-up point $[28,29]$. Further studies are required to determine the optimal check-up time point for reflecting postoperative NLR as a prognostic predictor in gastric cancer patients.

Table 3 Univariate and multivariate analyses of factors predicting disease-free survival

\begin{tabular}{|c|c|c|c|c|}
\hline & \multicolumn{2}{|l|}{ Univariate analysis } & \multicolumn{2}{|l|}{ Multivariate analysis } \\
\hline & $\mathrm{HR}(95 \% \mathrm{Cl})$ & $P$ value & Adjusted $\mathrm{HR}^{\mathrm{a}}(95 \% \mathrm{Cl})$ & $P$ value \\
\hline \multicolumn{5}{|l|}{ Preoperative NLR } \\
\hline LNLR $(N L R<2)$ & Reference & & Reference & \\
\hline HNLR (NLR $\geq 2)$ & $1.829(1.337-2.503)$ & 0.000 & $1.319(0.929-1.873)$ & 0.122 \\
\hline \multicolumn{5}{|l|}{ Postoperative NLR } \\
\hline LNLR (NLR < 1.7) & Reference & & Reference & \\
\hline HNLR (NLR $\geq 1.7)$ & $1.224(0.853-1.757)$ & 0.273 & $1.136(0.775-1.664)$ & 0.513 \\
\hline \multicolumn{5}{|l|}{ NLR change } \\
\hline LL (preoperative NLR < 2, postoperative NLR < 1.7) & Reference & & Reference & \\
\hline LH (preoperative NLR $<2$, postoperative NLR $\geq 1.7$ ) & $0.795(0.377-1.676)$ & 0.546 & $0.764(0.356-1.642)$ & 0.491 \\
\hline $\mathrm{HL}$ (preoperative NLR $\geq 2$, postoperative $N L R<1.7$ ) & $1.806(1.201-2.715)$ & 0.005 & $1.168(0.765-1.783)$ & 0.472 \\
\hline HH (preoperative NLR $\geq 2$, postoperative NLR $\geq 1.7$ ) & $1.955(1.269-3.011)$ & 0.002 & $1.400(0.892-2.197)$ & 0.143 \\
\hline
\end{tabular}


Table 4 Five-year overall survival based on tumor stage and the postoperative NLR

\begin{tabular}{|c|c|c|c|c|c|c|}
\hline & \multicolumn{2}{|l|}{ Stage I } & \multicolumn{2}{|l|}{ Stage II } & \multicolumn{2}{|l|}{ Stage III } \\
\hline & Number & 5 -YSR (\%) & Number & 5 -YSR (\%) & Number & 5 -YSR (\%) \\
\hline \multicolumn{7}{|l|}{ NLR } \\
\hline LNLR & 875 & 97.5 & 567 & 88.5 & 157 & 67.6 \\
\hline HNLR & 352 & 92.0 & 237 & 82.0 & 50 & 50.8 \\
\hline$P$ value & & 0.000 & & 0.241 & & 0.006 \\
\hline \multicolumn{7}{|l|}{ NLR change } \\
\hline LL (preoperative NLR $<2$, postoperative NLR $<1.7$ ) & 416 & 97.1 & 87 & 85.1 & 80 & 73.8 \\
\hline LH (preoperative NLR $<2$, postoperative NLR $\geq 1.7$ ) & 93 & 93.6 & 14 & 100.0 & 16 & 56.3 \\
\hline $\mathrm{HL}$ (preoperative NLR $\geq 2$, postoperative $\mathrm{NLR}<1.7$ ) & 150 & 98.7 & 68 & 92.7 & 71 & 60.6 \\
\hline $\mathrm{HH}$ (preoperative NLR $\geq 2$, postoperative NLR $\geq 1.7$ ) & 144 & 91.0 & 36 & 75.0 & 49 & 49.0 \\
\hline$P$ value & & 0.002 & & 0.034 & & 0.011 \\
\hline
\end{tabular}

5-YSR 5-year survival rate, NLR neutrophil-to-lymphocyte ratio, LNLR low NLR, HNLR high NLR, $L L$ low to low, $L H$ low to high, $H L$ high to low, HH high to high

\section{Conclusion}

Preoperative NLR, postoperative NLR, and changes in NLR were significant prognostic factors for OS in patients with gastric cancer. Patients with high preoperative and postoperative NLR, and especially with persistent elevation of preoperative to postoperative NLR, had poor prognosis with regard to OS. Based on these results, we suggest that the NLR should be included in the routine postoperative assessment of patients with gastric cancer. The identification of high-risk patients will allow early interventions that may reduce the risk of recurrence and improve oncological outcomes.

\section{Supplementary Information}

Supplementary information accompanies this paper at https://doi.org/10. 1186/s12957-020-02059-4.

Additional file 1: Table S1. Associations of patient characteristics with the postoperative neutrophil-to-lymphocyte ratio (NLR).

\section{Acknowledgements}

The authors have no conflicts of interest or financial ties to disclose. Statistical consultation was supported by the Department of Biostatistics of the Catholic Research Coordinating Center. The English in this document has been checked by at least two professional editors, both native speakers of English. For a certificate, please see:

http://www.textcheck.com/certificate/3FCXv5

\section{Authors' contributions}

Eun Young Kim and Kyo Young Song designed the research; Eun Young Kim and Kyo Young Song performed the research; Eun Young Kim and Kyo Young Song analyzed the data; Eun Young Kim and Kyo Young Song wrote the paper. The author(s) read and approved the final manuscript.

\section{Funding}

None.

\section{Availability of data and materials}

Access to the database may be obtained from the corresponding author on reasonable request.
Ethics approval and consent to participate

The study was approved by the Human Experimentation Committee of our institution (ethical approval number: KC17RESI0108).

\section{Consent for publication}

We simply extracted data and did not involve the private information of patients.

\section{Competing interests}

The authors declare that they have no competing interests.

\section{Author details}

'Department of Surgery, Uljeongbu St. Mary Hospital, College of Medicine, The Catholic University of Korea, Seoul, Korea. ${ }^{2}$ Department of Surgery, Seoul St. Mary's Hospital, College of Medicine, The Catholic University of Korea, Seoul, Korea.

Received: 5 August 2020 Accepted: 20 October 2020

Published online: 10 November 2020

References

1. Torre LA, Bray F, Siegel RL, Ferlay J, Lortet-Tieulent J, Jemal A. Global cancer statistics, 2012. CA Cancer J Clin. 2015;65:87-108.

2. Japanese Gastric Cancer Association: Japanese gastric cancer treatment guidelines 2014 (ver. 4). Gastric Cancer 2017:20:1-19.

3. Diakos Cl, Charles KA, McMillan DC, Clarke SJ. Cancer-related inflammation and treatment effectiveness. Lancet Oncol. 2014;15:e493-503.

4. Kang M, Jeong CW, Kwak C, Kim HH, Ku JH. The prognostic significance of the early postoperative neutrophil-to-lymphocyte ratio in patients with urothelial carcinoma of the bladder undergoing radical cystectomy. Ann Surg Oncol. 2016;23:335-42.

5. Kim YH, Choi WJ. The effectiveness of postoperative neutrophils to lymphocytes ratio in predicting long-term recurrence after stomach cancer surgery. J Korean Surg Soc. 2012;83:352-9.

6. Dan J, Zhang Y, Peng Z, Huang J, Gao H, Xu L, Chen M. Postoperative neutrophil-to-lymphocyte ratio change predicts survival of patients with small hepatocellular carcinoma undergoing radiofrequency ablation. PLoS One. 2013;8:e58184

7. Li C, Wen TF, Yan LN, Li B, Wang WT, Yang JY, Xu MQ. Postoperative neutrophil-to-lymphocyte ratio plus platelet-to-lymphocyte ratio predicts the outcomes of hepatocellular carcinoma. J Surg Res. 2015:198:73-9.

8. Ohno Y, Nakashima J, Ohori M, Gondo T, Hatano T, Tachibana M. Followup of neutrophil-to-lymphocyte ratio and recurrence of clear cell renal cell carcinoma. J Urol. 2012;187:411-7.

9. Miyatani K, Saito H, Kono Y, Murakami Y, Kuroda H, Matsunaga T, Fukumoto Y, Osaki T, Ashida K, Fujiwara Y. Combined analysis of the pre- and postoperative neutrophil-lymphocyte ratio predicts the outcomes of patients with gastric cancer. Surg Today. 2018;48:300-7. 
10. Min KW, Kwon MJ, Kim DH, Son BK, Kim EK, Oh YH, Wi YC. Persistent elevation of postoperative neutrophil-to-lymphocyte ratio: a better predictor of survival in gastric cancer than elevated preoperative neutrophil-tolymphocyte ratio. Sci Rep. 2017;7:13967.

11. Tanaka H, Tamura T, Toyokawa T, Muguruma K, Miki Y, Kubo N, Sakurai K, Hirakawa K, Ohira M. Clinical relevance of postoperative neutrophillymphocyte ratio (NLR) to recurrence after adjuvant chemotherapy of S-1 for gastric cancer. Anticancer Res. 2018;38:3745-51.

12. Kim EY, Lee JW, Yoo HM, Park CH, Song KY. The platelet-to-lymphocyte ratio versus neutrophil-to-lymphocyte ratio: which is better as a prognostic factor in gastric cancer? Ann Surg Oncol. 2015;22:4363-70.

13. Lin JX, Wang ZK, Huang YQ, Xie JW, Wang JB, Lu J, Chen QY, Lin M, Tu RH, Huang ZN, et al: Dynamic changes in pre- and postoperative levels of inflammatory markers and their effects on the prognosis of patients with gastric cancer. 2020

14. Miyamoto R, Inagawa S, Sano N, Tadano S, Adachi S, Yamamoto M. The neutrophil-to-lymphocyte ratio (NLR) predicts short-term and long-term outcomes in gastric cancer patients. Eur J Surg Oncol. 2018;44:607-12.

15. Yu L, Lv CY, Yuan AH, Chen W, Wu AW. Significance of the preoperative neutrophil-to-lymphocyte ratio in the prognosis of patients with gastric cancer. World J Gastroenterol. 2015;21:6280-6.

16. Graziosi L, Marino E, De Angelis V, Rebonato A, Cavazzoni E, Donini A. Prognostic value of preoperative neutrophils to lymphocytes ratio in patients resected for gastric cancer. Am J Surg. 2015;209:333-7.

17. Zhou D, Wu Y, Zhu Y, Lin Z, Yu D, Zhang T. The prognostic value of neutrophil-to-lymphocyte ratio and monocyte-to-lymphocyte ratio in metastatic gastric cancer treated with systemic chemotherapy. I Cancer. 2020;11:4205-12

18. Hirahara T, Arigami T. Combined neutrophil-lymphocyte ratio and plateletlymphocyte ratio predicts chemotherapy response and prognosis in patients with advanced gastric cancer. BMC Cancer 2019;19:672.

19. Mantovani A, Allavena P, Sica A, Balkwill F. Cancer-related inflammation. Nature. 2008;454:436-44.

20. Grivennikov SI, Greten FR, Karin M. Immunity, inflammation, and cancer. Cell. 2010;140:883-99.

21. Coussens LMWZ. Inflammation and cancer. Nature. 2002;420:860-7.

22. Powell DR, Huttenlocher A. Neutrophils in the tumor microenvironment. Trends Immunol. 2016;37:41-52.

23. De Palma M, Biziato D, Petrova TV. Microenvironmental regulation of tumour angiogenesis. Nat Rev Cancer. 2017;17:457-74.

24. Gonzalez $\mathrm{H}$, Hagerling C, Werb Z. Roles of the immune system in cancer: from tumor initiation to metastatic progression. Genes Dev 2018;32:126784.

25. Jang WS, Cho KS, Kim MS, Yoon CY, Kang DH, Kang YJ, Jeong WS, Ham WS, Choi YD. The prognostic significance of postoperative neutrophil-tolymphocyte ratio after radical prostatectomy for localized prostate cancer. Oncotarget. 2017;8:11778-87.

26. Marrelli D, Pinto E, De Stefano A, Farnetani M, Garosi L, Roviello F. Clinical utility of CEA, CA 19-9, and CA 72-4 in the follow-up of patients with resectable gastric cancer. Am J Surg. 2001;181:16-9.

27. Dabrowska AM, Slotwinski R. The immune response to surgery and infection. Cent Eur J Immunol. 2014;39:532-7.

28. Jin F, Han A, Shi F, Kong L, Yu J. The postoperative neutrophil-tolymphocyte ratio and changes in this ratio predict survival after the complete resection of stage I non-small cell lung cancer. Onco Targets Ther. 2016;9:6529-37.

29. Josa V, Krzystanek M, Eklund AC, Salamon F, Zarand A, Szallasi Z, Baranyai Z. Relationship of postoperative thrombocytosis and survival of patients with colorectal cancer. Int J Surg. 2015;18:1-6.

\section{Publisher's Note}

Springer Nature remains neutral with regard to jurisdictional claims in published maps and institutional affiliations.

Ready to submit your research? Choose BMC and benefit from:

- fast, convenient online submission

- thorough peer review by experienced researchers in your field

- rapid publication on acceptance

- support for research data, including large and complex data types

- gold Open Access which fosters wider collaboration and increased citations

- maximum visibility for your research: over $100 \mathrm{M}$ website views per year

At $\mathrm{BMC}$, research is always in progress.

Learn more biomedcentral.com/submissions 